\title{
The Role of Noise in Determining Selective Ionic Conduction Through Nano-Pores
}

\author{
W.A.T. Gibby*, M.L. Barabash*, C. Guardiani*, D.G. Luchinsky*† and P.V.E. McClintock* \\ *Department of Physics \\ Lancaster University, Lancaster, UK, LA1 4YB \\ Email: w.gibby@lancaster.ac.uk \\ ${ }^{\dagger}$ SGT Inc., Greenbelt MD, 20770, USA \\ Email: d.luchinsky@lancaster.ac.uk
}

\begin{abstract}
The problem of predicting selective transport of ions through nano-pores from their structure in the biological and nano-technological systems is addressed. We use a molecular dynamics simulation to provide insight into the key physical parameters of nano-pores and develop a self-consistent analytic theory describing ionic conduction and selectivity through these devices. We analyse the ion's dehydration and excess chemical potential, derive an expression for the conductivity of the nanopore, and emphasize the role of fluctuations in its performance. The theory is verified by comparison of the predicted currentvoltage characteristics with the molecular dynamics results and experimental data obtained for a graphene nano-pore and the KesA biological channel.
\end{abstract}

Index Terms-Graphene nanopore, biological ion channel, selectivity, functionalisation, molecular dynamics, statistical theory, kinetic theory.

\section{INTRODUCTION}

The investigation of ionic transport through nano-pores is a vast and rapidly expanding field encompassing both the nano and bio-physics communities. These pores have radii on sub-nanometer length scales and can be as thin as a single layer of graphene; and yet their selectivity properties are difficult to predict and/or control. Interest in these pores is stimulated both by the wide range of applications including e.g. the desalination of water [1], and by the novel light they shed on understanding the function of biological ion channels [2]. In particular, the bio-inspired functionalisation of these nano-pores offers a challenging avenue for the composition of nano-scale devices with pre-defined properties. Another long-standing problem in the analysis of these devices is the development of an analytic theory to describe the trade off between their high selectivity and fast permeability [3]-[5].

In this work we aim to address these challenges by introducing a self-consistent approach to investigating the effect of hydration and fluctuations on the performance of nano-pores. We develop statistical and kinetic theories of their conductivity and selectivity. Theoretical predictions are compared with experimental and simulation data of conduction through a single-layer graphene pore and a biological channel KcsA.

\section{ION PERMEATION THROUGH A PORE IN GRAPHENE}

In a preliminary simulation study we have compared the properties of a neutral and a charged pore opened inside a single graphene sheet. The aim of these simulations is to highlight the most important factors affecting ion permeation and investigate the role of hydration shells. Both charged and neutral systems (left panel on Fig. 1) comprise a single fixed
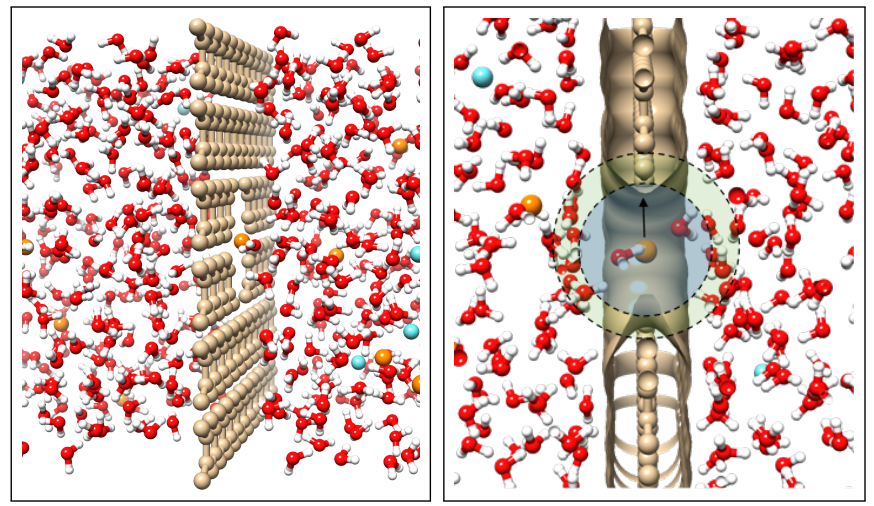

Fig. 1. Illustration of the simulation set-up. The graphene layer is shown by the golden sheet and potassium and chloride ions are represented by orange and cyan spheres respectively. Water molecules are shown in red (oxygen) and white (hydrogen). (Left) Side view. (Right) Pore cross-section with depicted first (transparent blue) and second (transparent green) hydration shells and hydrophobic surface.

graphene layer with a pore hydrated on both sides by a $1 \mathrm{M}$ $\mathrm{KCl}$ solution. The minimal carbon-carbon distance in the pore is $\sim 0.45 \mathrm{~nm}$, and so the effective pore radius accounting for the hydrophobicity is $\sim 0.23 \mathrm{~nm}$. The dimension of the simulation box is $2.6 \times 2.4 \times 4 \mathrm{~nm}$, and following Ref. [6] the geometry of the pore was chosen to match the shape of one of the four carbonyl rings in the selectivity filter (SF) of KcsA channel. To create the charged system, four $\mathrm{C}$ atoms on the pore rim were assigned charges $-0.5 q$ (matching the oxygen of the carbonyl groups), and the electro-neutrality of the system was enforced by a balancing charge of $+2.0 q$ uniformly spread over all the remaining graphene atoms.

In order to obtain the potential of mean force (PMF) of the permeating potassium ion $\left(\mathrm{K}^{+}\right)$the umbrella sampling method was employed. In these simulations the axial coordinate $z$ of a single $\mathrm{K}^{+}$was harmonically restrained while the radial position was subject to a semi-harmonic wall at $0.45 \mathrm{~nm}$ from the pore axis. We considered 61 axial windows from $z=-1.5 \mathrm{~nm}$ to $1.5 \mathrm{~nm}$ with width of $0.05 \mathrm{~nm}$. Each window was run for $1.5 \mathrm{~ns}$ with the first $0.5 \mathrm{~ns}$ considered as equilibration and excluded from the analysis. Using the implementation by Grossfield [7] the weighted histogram analysis method (WHAM) [8] was used to calculate the PMF with a tolerance of $10^{-7}$.

Both the charged and neutral PMF are highlighted in Fig. 2 as red and blue respectively. The neutral pore produces a barrier of $7.31 k T$, whilst the charged pore yields a barrier 

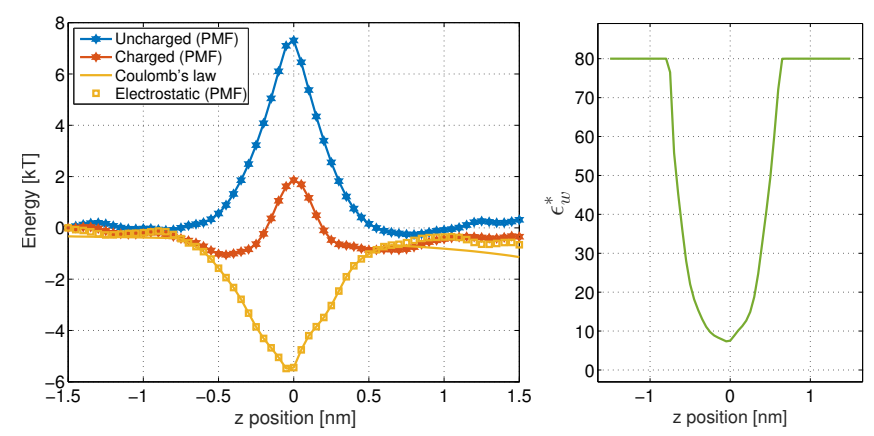

Fig. 2. (Left) Potential of Mean Force (PMF) of potassium in the neutral (blue stars) and charged (red stars) graphene systems. Orange squares represent the difference between those PMFs yielding the electrostatic energy contribution. (Right) Dielectric permittivity variation on the way through the pore.

of $2.81 k T$ agreeing with a similar set up as reported by Sahu et al. [9], and He et al. [6] for a 4CO system. This suggests that the height of the barrier is primarily determined by the charge of the pore and not by the specific geometry or placement of the functional groups. The fact that this barrier is much smaller than the one computed for the neutral system confirms that the presence of negative charges in the pore favours cation permeation. The PMF in Fig. 2 also shows the existence of two minima on either side of the graphene monolayer, centered at $z=-0.5 \mathrm{~nm}$ and $+0.5 \mathrm{~nm}$. The two free energy minima arise because in these locations the $\mathrm{K}^{+}$ is close enough to the pore to feel the electrostatic attraction of the charges but it is still not in the gap region between the water phase and the graphene layer, so it is still not significantly dehydrated.

The electrostatic component $G_{C}$ of the charged PMF is related to the Coulomb potential between $n_{f}$ negative pore charges, and the ion $z q$ : $G_{C}=z q^{2} n_{f} / 4 \pi \epsilon_{w}^{*} \epsilon_{0} R_{i f}$. (Hereafter, $q$ is the elementary charge, $\epsilon_{0}$ is vacuum permittivity, and $R_{i f}$ is the distance between ion and pore charge.) Therefore, subtracting the neutral PMF from the charged one, we can find the effective local permittivity of water $\epsilon_{w}^{*}$ (see right panel in Fig. 2), which decreases as the ion approaches the pore, taking the average value $\sim 9$ inside. This indicates that water in the pore should not be treated as in the bulk, and can be explained by noting that, despite the minor dehydration (see subsequent discussion and the right panel of Fig. 1), the majority of the charge-screening water molecules in the shells reside outside of the pore.

To investigate the role of hydration in more details, we have computed the number of water molecules in each hydration shell surrounding a restrained $\mathrm{K}^{+}$ion in the windows of the umbrella sampling simulations. As shown in Fig. 3, both the first and the second hydration shell lose water upon crossing the pore but the depletion is more significant for the second shell, in agreement with the results of Sahu et al. [9]. This occurs because, when the $\mathrm{K}^{+}$ion crosses the pore, it tends to stay close to the charged carbon atoms establishing direct salt bridges, thus minimising the available space for coordinating waters. On the other hand, the water molecules of the second shell lying further outside the pore are still attracted electrostatically to the charged rim as well as the ion, and thus still remain in the neighborhood of the ion.
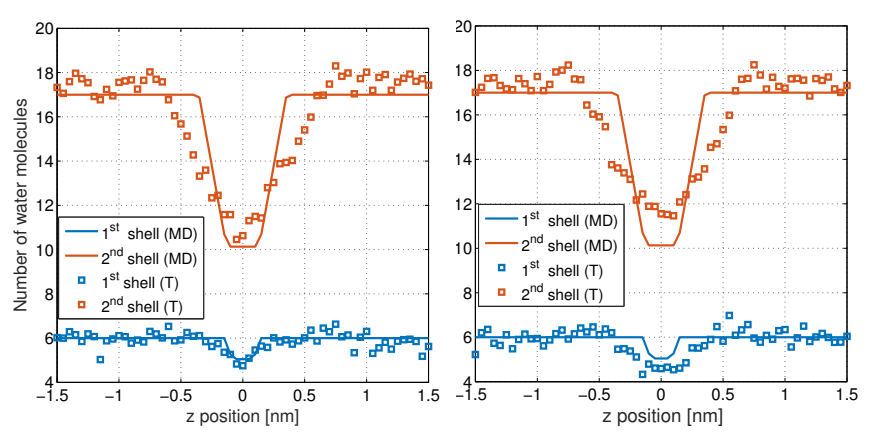

Fig. 3. Potassium hydration profiles in the neutral (left) and charged (right) graphene systems. Squares represent the number of water molecules obtained from MD for the first (blue) and second (orange) coordinating shells. Theoretical predictions of the dehydration fraction $f_{j}$ are given by solid lines.

For analytic calculations one needs to estimate the dehydration energy for each shell. To do so, Zwolak and coworkers [10] proposed to couple the dehydration energy and the cost for its change with shell's surface area. Thus, the energy barrier becomes a function of the pore radius $R_{c}$ : $\Delta U\left(R_{c}\right)=\sum_{j}\left(f_{j}\left(R_{c}\right)-1\right) U_{j}^{0}$. Here the sum runs over all $j$ shells, and the dehydration fraction $f_{j}$ is the remaining portion of shell $j$ left upon permeation through a pore of radius $R_{c}$. The energy $U_{j}^{0}$ stored in each hydration shell is

$$
U_{j}^{0}=\frac{z q^{2}}{8 \pi \epsilon_{0}}\left(\frac{1}{\epsilon_{p}}-\frac{1}{\epsilon_{w}}\right)\left(\frac{1}{R_{j}^{0}}-\frac{1}{R_{j}^{I}}\right),
$$

where $R_{j}^{0}$ and $R_{j}^{I}$ are the outer and inner radii of the shell respectively, and $\epsilon_{p}$ is the permittivity of the membrane. The solid lines on Fig. 3 display this fraction for $R_{c}=0.23 \mathrm{~nm}$ and length $L_{c}=0.4 \mathrm{~nm}$. As long as the fraction is computed solely from the geometry, the dehydration profiles (1) are identical for both the neutral and charged systems. Although the estimation (1) lacks water-water and water-fixed charge interactions, one sees quite good agreement particularly for the neutral pore. The differences can be attributed in part due to the lack of these interactions, but also the influence of nearby ions and the cylindrical approximation for the pore geometry.

The above results justify the picture that $\mathrm{K}^{+}$ion outside the pore is not significantly dehydrated and hence the strong electrostatic attraction it faces produces the two free energy minima flanking the barrier in the PMF (Fig. 2). This interpretation is consistent with Fig. 3 where in positions $\pm 0.5 \mathrm{~nm}$ the first hydration shell is practically intact while the second shell has only lost approximately $40 \%$ of its water molecules.

Thus, the results of our preliminary MD simulations indeed identify two driving forces for ion permeation: (i) the ionpore electrostatic interactions and (ii) the dehydration cost the ion incurs when crossing the pore. This is in agreement with the seminal theory developed by Eisenman [3]. In the next section, we incorporate those energy contributions in statistical theory.

\section{THEORY OF SELECTIVE CONDUCTION OF MOLECULAR PORE: ROLE OF FLUCTUATIONS}

The preceding discussion demonstrates that, to take explicit account of dehydration and strong interaction between conducting and fixed ions at the pore, a self consistent theory 
has to be defined at the molecular level. We note further that under typical experimental conditions the thermal de Broglie wavelength $\Lambda_{i}$ of ions of $i$-th type in the solution is of the order of $\sim 0.03 \mathrm{~nm}$. This is much less than the average distance $\left(\left(V / n_{i}\right)^{1 / 3}\right)$ between ions in the bulk and even in narrow pores and so the system is classical and the ions obey Boltzmann statistics. Therefore the physical properties of the whole system, comprising a pore and two bulk reservoirs, are determined by the canonical ensemble assuming that it conserves total volume $V$, temperature $T$ and the total number of particles of each species $N_{i}$. The total energy of this ensemble has the form [11]-[13]

$$
\begin{aligned}
E\left(\left\{n_{j}\right\}, n_{f}\right) & =E_{0}+\left(N_{w}-n_{w}\right) \bar{\mu}_{w}^{b}+n_{w} \bar{\mu}_{w}^{c} \\
& +\sum_{i}\left[\left(N_{i}-n_{i}\right) \mu_{i}^{b}+n_{i} \mu_{i}^{c}\right] \\
& -k T \ln \left(\frac{K !}{n_{w} !} \prod_{i} \frac{1}{n_{i} !}\right)+\mathcal{E}\left(\left\{n_{j}\right\}, n_{f}\right) .
\end{aligned}
$$

Here, $\left\{n_{j}\right\}$ is a set of ions in the pore, $E_{0}=T S-p V$ is the thermodynamic part of system's energy for a given entropy $S$ and pressure $p$. The next two terms in the first row explicitly account for the energy changes due to the transfer of the water molecules between bathing solutions and the pore.

The second row describes the energy required to add $n_{i}$ ions of the $i$-th type to the pore. In the last row the first term accounts for permutations of ions of each type and water molecules, while $\mathcal{E}\left(\left\{n_{j}\right\}, n_{f}\right)$ corresponds to ion-ion and ionfixed charge interactions inside the pore. The electrochemical potential of the ions is $\mu_{i}^{b, c}=\mu_{0, i}^{b, c}+\tilde{\mu}_{i}^{b, c}$, where $\mu_{0, i}^{b, c}$ represents its ideal part and $\tilde{\mu}_{i}^{b, c}=\bar{\mu}_{i}^{b, c}+q \phi^{b, c}$ consists of the electric $\phi^{b, c}$ and excess chemical $\bar{\mu}_{i}^{b, c}$ potentials in the bulk $(b)$ or in the pore $(c)$. In what follows we neglect the difference in the thermal wavelength and the internal degrees of freedom between the bulk and the pore, and the effect of electric field on the motion of water molecules because $z_{w} \sim 0$.

The single-file conduction of ions through the pore with a finite number of binding sites $K$ defines the set of energy states of the pore and its total occupancy [12]-[17]. Observing that transitions of ions from the bulk to the pore correspond to small fluctuations in the total energy of the system [11] and canceling out constant terms in the energy Eq. (2) we obtain [12], [13] the free energy of such transitions

$$
\begin{aligned}
G\left(\left\{n_{j}\right\}, n_{f}\right)= & \mathcal{E}\left(\left\{n_{j}\right\}, n_{f}\right)+\sum_{i} n_{i}\left[\tilde{\mu}_{i}^{c}-\tilde{\mu}_{i}^{b}\right] \\
& -k T \ln \left(\frac{1}{n_{w} !} \prod_{i} \frac{\left(x_{i}^{b}\right)^{n_{i}}}{n_{i} !}\right) .
\end{aligned}
$$

The corresponding grand-canonical ensemble reads

$$
P\left(\left\{n_{j}\right\}, n_{f}\right)=\frac{1}{\mathcal{Z} n_{w} !} \prod_{i} \frac{\left(x_{i}^{b}\right)^{n_{i}}}{n_{i} !} e^{\frac{\sum_{i} n_{i}\left[\tilde{\mu}_{i}^{c}-\tilde{\mu}_{i}^{b}\right]-\mathcal{E}\left(\left\{n_{j}\right\}, n_{f}\right)}{k T}}
$$

where the grand partition function $\mathcal{Z}$ is defined from the normalisation condition $\sum_{\left\{n_{j}\right\}} P\left(\left\{n_{j}\right\}, n_{f}\right) \equiv 1$, and yields the grand potential

$$
\Omega=-k T \ln \mathcal{Z}
$$

Thus we arrive at a statistical model of the pore that takes explicit account of dehydration (via $\bar{\mu}_{i}$ ) and strong interaction (via $\mathcal{E}$ ) between ions inside the pore.

In order to estimate $\bar{\mu}_{i}$ and $\mathcal{E}$, and to introduce their analytic approximations, we notice that the probabilities $P\left(\left\{n_{i}\right\}, n_{f}\right)$ for a given configuration of ions $n_{i}$ in the pore are related to the binding probabilities $\mathcal{B}\left(\left\{n_{j}\right\}, n_{f}\right) \sim$ $P\left(\left\{n_{i}\right\}, n_{f}\right) / P\left(\{0\}, n_{f}\right)$ introduced by Roux [18], [19] leading to,

$$
e^{-\frac{\left(\sum_{i} n_{i}\left[\bar{\mu}_{i}^{c}-\bar{\mu}_{i}^{b}\right]+\mathcal{E}\left(\left\{n_{j}\right\}, n_{f}\right)\right)}{k T}} \sim \int e^{-\frac{\mathcal{W}\left(\mathbf{r}_{1}, \ldots, \mathbf{r}_{n_{i}}\right)-n_{i} \bar{\mu}_{i}^{b}}{k T}} d \mathbf{r}^{n_{i}}
$$

with $\mathcal{W}\left(\mathbf{r}_{1}, \ldots, \mathbf{r}_{n_{i}}\right)$ standing for the $n$-ion PMF of the pore and the integration runs over the pore volume. The analysis in Sec. II shows that the main contributions into the $n$-ion PMF are due to dehydration Eq. (1) [10] and to the local interactions between the mobile ion and surrounding charges. The latter can be approximated by electrostatic interactions with effective dielectric permittivity of the pore.

In addition, the global ion-ion interaction in a charged water-filled pore can be approximated analytically [15], [17], [20] using the notion of the charging energy of the pore familiar from the Coulomb blockade theory of quantum dots, see e.g. [21], [22]. The resulting expression is

$$
\mathcal{E}\left(\left\{n_{j}\right\}, n_{f}\right)=\frac{q^{2} L_{c}}{8 \pi \epsilon_{0} \epsilon_{w} R_{c}^{2}}\left(n_{f}+\sum_{i} z_{i} n_{i}\right)^{2}
$$

where the prefactor ${ }^{1}$, can be denoted by $U_{c}=q^{2} / 2 C_{s}$ with the pore capacitance being $C_{s}$.

\section{SElectivity VS CONDUCTIVITY TRADE-OFF}

The current density $j_{i}$ through the pore can be written in general form [19], [23]-[25] as $j_{i}=-\frac{\sigma_{i}}{q} \nabla \mu_{i}$, where the conductivity $\sigma$ is defined using the generalised Einstein relation [12], [26], [27]

$$
\sigma_{i}=q^{2} D_{i} \frac{\partial c_{i}}{\partial \eta_{i}} \propto\left\langle\Delta n_{i}^{2}\right\rangle=-k T\left(\frac{\partial^{2} \Omega}{\partial \eta_{i}^{2}}\right)_{T, V},
$$

where $c_{i}=\left\langle n_{i}\right\rangle / V_{c}$ is the concentration, $D_{i}$ is the diffusivity and $\eta_{i}$ is the chemical potential in the pore with volume $V_{c}$. The current is directly proportional to the fluctuations in the number of ions and, accordingly, current is largest when the rate of these fluctuations is maximal.

In the particular case of a three level system $\{0\},\{X\}$, $\{Y\}$, where the ground state 0 corresponding to a given set $\left\{n_{j}\right\}$ of ions in the system is excited by adding an ion of type $X$ or $Y$ to the pore, the conductivity of each ion type is

$$
\left\langle\Delta n_{X}^{2}\right\rangle=\frac{e^{-\Delta G_{X} / k T}+e^{-\left(\Delta G_{Y}+\Delta G_{X}\right) / k T}}{\left(1+e^{-\Delta G_{X} / k T}+e^{-\Delta G_{Y} / k T}\right)^{2}}
$$

where $\Delta G_{i}=G\left(n_{i}+1, n_{f}\right)-G\left(n_{i}, n_{f}\right)$ stands for the free energy barriers and an analogous expression for $Y$ is obtained by exchanging symbols $X$ and $Y$.

It follows from equation (9) that maximum conductivity for a given type of ions (e.g. $X$ ) is achieved when

$$
\Delta G_{X} \sim 0 \text {, and } \Delta G_{X} \ll \Delta G_{Y},
$$

${ }^{1}$ This charging paramater reflects the strength of interaction between the mobile ions and the pore charge. 
hold simultaneously. The first relation represents the condition for barrier-less conduction. Given that transitions occur between $N$ and $N+1$ states, one recognises the knockon mechanism. Eventually, the last condition embodies the strong selectivity regime. Thus, maximising the noise (variance) of pore occupancy, one derives the optimal conduction and selectivity properties. We note that it is surprising that exactly these conditions are found in Nature, namely in the biological KcsA channel.

Thus the optimal parameters of the nano-pore for selective conduction at nearly the diffusion rate can be now obtained analytically in the form

$$
\begin{aligned}
n_{f}^{*} & =\frac{C_{s}}{q^{2}}\left(\Delta \mu_{X}^{*}-k T \ln \left[\frac{\left(n_{X}+1\right)}{\left(K-n_{X}-n_{Y}\right)}\right]\right) \\
& -\left(n_{X}+\frac{1}{2}\right),
\end{aligned}
$$

the optimal chemical potential difference $\left(\Delta \mu_{X}^{*}=\mu_{X}^{b}-\mu_{X}^{c}\right)$ is given by

$$
\Delta \mu_{X}^{*}=\frac{1}{n_{X}} \mathcal{E}\left(n_{X}, n_{f}^{*}\right)+\frac{k T}{n_{X}} \ln \left[\left(n_{X}\right) !\left(K-n_{X}-n_{Y}\right) !\right] .
$$

It is clear therefore that the pore allows for the unhindered (but still diffusion-limited) passage of these favoured ions, while selectively blocking the other species. The pore is tuned to satisfy this condition, resulting in the following energy barrier for an ion of the same valence

$$
\begin{aligned}
\Delta \Delta G & =\left(\bar{\mu}_{Y}^{c}-\bar{\mu}_{Y}^{b}\right)-\left(\bar{\mu}_{X}^{c}-\bar{\mu}_{X}^{b}\right)+k T \ln \frac{c_{X}}{c_{Y}} \\
& +k T \ln \frac{n_{Y}+1}{n_{X}+1} .
\end{aligned}
$$

Provided that the free energy barrier for each type of ion to enter the pore Eq. (3) is a global function of the pore parameters, for ions of the same valence the difference between these barriers is determined mainly by the local interactions in the pore as envisaged by Eisenman [3]. This result is intuitively reasonable since on distances of a few ionic radii, the ions behave as point changes and cannot be distinguished. The above analysis is equally applicable for uncharged pores, different pore radii or lengths and ions of different valence.

Hence, we have shown that a generalised form (12) of Eisenman's selectivity relation follows directly from the condition for the diffusion-limited conduction of $\mathrm{K}^{+}$ions. The corresponding conductivity of the favoured ion can now be written as

$$
\sigma_{X}=\frac{q^{2} D_{X} e^{-\Delta G_{X} / k T}}{V_{c} k T\left(1+e^{-\Delta G_{X} / k T}\right)^{2}},
$$

\section{KINETIC THEORY}

To investigate non-equilibrium responses we will introduce a self-consistent kinetic theory, describing permeation with rate $\Gamma$ through the discrete state space identified in the previous section. The behavior can be analysed with a discrete set of master equations, where the transiting ion is denoted by $n_{i}^{*}$,

$$
\dot{P}\left(\left\{n_{j}\right\}\right)=-\sum_{i} \sum_{b} \sum_{J=\left\{\begin{array}{c}
n_{j}+n_{i}^{*} \\
n_{j}-n_{i}^{*} \\
\star
\end{array}\right\}} P\left(\left\{n_{j}\right\}\right) \Gamma_{n_{j}, J}^{b, i}-P(\{J\}) \Gamma_{J, n_{j}}^{b, i} .
$$

The left hand side of the equation delineates the temporal behavior and must equal zero in the steady-state regime. The sums run over species $i$, both left and right bulks $b$, and for clarity conditions $\star$. These conditions require that the state of the pore must remain within the state space and the transitions be physically possible i.e. the number of ions cannot exceed the number of sites, and an ion can only exit if it is already occupying the pore. Formally this can be defined as $0 \leq J \leq$ $K-n_{w}$ and when removing an ion $n_{j}-n_{i}^{*} \neq \sum_{i^{\prime} \neq i} n_{i^{\prime}}-n_{i}^{*}$ where $\sum_{i^{\prime} \neq i} n_{i^{\prime}}$ denotes the sum over all ions not including the transiting $i^{\text {th }}$ species. For notational clarity we drop the functional dependence on $n_{f}$ although it remains present in the energy barrier.

The steady-state current can be defined as in standard practice from the balance of fluxes, such that Kirchoff's laws are satisfied. It is defined such that its positivity defines ionic flow from left to right,

$I_{i}^{b}= \pm q \sum_{n_{j}=0}^{K-1-n_{w}} P\left(\left\{n_{j}\right\}\right) \Gamma_{n_{j}, n_{j}+n_{i}^{*}}^{b, i}-P\left(\left\{n_{j}+n_{i}^{*}\right\}\right) \Gamma_{n_{j}+n_{i}^{*}, n_{j}}^{b, i}$.

The transition rates are defined such that at equilibrium the detailed balance conditions equate to the Boltzmann ratio with the equilibrium free energy barrier given by equation (3). The energy profile is assumed to take the form of a binding site whereby the particle must escape and therefore the influence from the potential drop is assumed to be solely inside the pore [28]. We note that this can be extended to include a chemical gradient if needed. The rates therefore take the following form,

$$
\begin{aligned}
& \Gamma_{n_{j}+n_{i}^{*}, n_{j}}^{b, i}=A_{i} e^{\left(\Delta G_{i}-z_{i} q\left(\phi^{b}-\phi^{c}\right)\right) / k T}, \\
& \Gamma_{n_{j}, n_{j}+n_{i}^{*}}^{b, i}=A_{i}, \quad \text { where: } A_{i}=\frac{2 D_{i}}{L_{c}^{2}\left(1+e^{\Delta G_{i} / k T}\right)} .
\end{aligned}
$$

The equilibrium normalisation $A_{i}$ must relate to the general energy profile of the system and in its linear response must reproduce Ohm's and Fick's laws for the favoured ion as calculated earlier in equation (13). It is important to note, however, that we do not include a transition state and nor do we explicitly track the motion of ions through sites. These developments will be considered in further publications.

\section{COMPARISON WITH EXPERIMENTAL DATA}

\section{A. Example 1: Graphene nanopore}

The results from $\mathrm{He}$ et al. [6] provide an excellent test of our theory, because the authors simulate a set of charged graphene nanopores which are similar to the selectivity filters of biological channels. The pore diameters are comparable to these filters although the length is smaller $\sim 0.4 \mathrm{~nm}$; meanwhile different charges are added with the intention of reproducing $\mathrm{K}^{+}$or $\mathrm{Na}^{+}$selectivity. These charges are either carbonyl groups with a negative partial oxygen charge or 


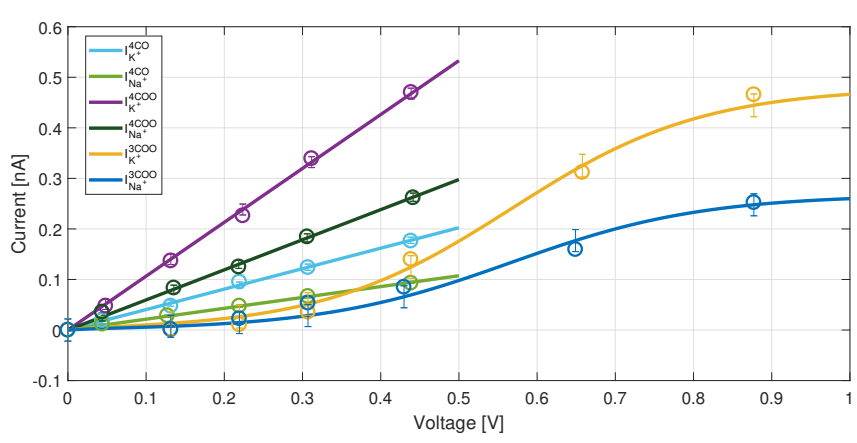

Fig. 4. Comparison of theoretical current (solid lines) to simulated conduction through single-layer graphene (circles) [6]. The diffusion coefficient in the pore is found to be smaller than in the bulk $0.125 D_{i}^{b}$, as expected in narrow pores [29]. The linear responses were fit against equation (13) with free energy barriers: $3.02 k T$ and $3.28 k T$ for $\mathrm{K}^{+}$and $\mathrm{Na}^{+}$in $4 \mathrm{CO}$ and $-3.51 k T$ and $-1.38 k T$ for $\mathrm{K}^{+}$and $\mathrm{Na}^{+}$in 4COO. The non-ohmic current through $3 \mathrm{COO}$ was fit against our equation (15) and the energy barriers were $-3.85 k T$ and $-1.41 k T$ for $\mathrm{K}^{+}$and $\mathrm{Na}^{+}$respectively and the pore was highly asymmetrical with the binding site experiencing $\sim 1 / 5$ of the voltage drop $\left(\phi^{c} \sim \phi^{L} / 5\right)$

carboxylate groups with a permanent charge of $-1 q$. We shall consider all three pores: the four carbonyl (4CO), four carboxylate (4COO) and three carboxylayte (3COO). Both $4 \mathrm{CO}$ and $4 \mathrm{COO}$ demonstrate linear responses and so are compared with our linearised expression of current (13); and so the non-Ohmic $3 \mathrm{COO}$ pore is compared with our kinetic equation (15). The system was set up with constant temperature $300 \mathrm{~K}$ and mixed $\mathrm{KCl}$ and $\mathrm{NaCl}$ solutions of $0.5 \mathrm{M}$ each, and $\mathrm{Cl}^{-}$does not permeate the pore. Thus we can consider the system as having one binding site, and competing single cation permeation because the charging parameter is relatively small $U_{c} \sim 9$ to $27 k T$ when an averaged value in the pore of $\epsilon_{w}^{*}=9$ is used. Fitting is provided in Fig. 4 with the fitting parameters given in the caption; and we note that the diffusion coefficient in the pore is smaller than in the bulk as expected [29]. In general the fitting is good with a small residual standard error for each data set, which are given as error bars.

\section{B. Example 2: $\mathrm{K}^{+}$biological ion channel (KcsA)}

KcsA is a bacterial $\mathrm{K}^{+}$ion channel strongly favouring $\mathrm{K}^{+}$over $\mathrm{Na}^{+}$at ratios of 1000:1 [2]. First crystallised in 1998 [30], it represents a model ion channel because of its shared homology with other $\mathrm{K}^{+}$channels particularly within the narrow selectivity filter. We approximate this filter as a cylinder ${ }^{2}$ of length $1.2 \mathrm{~nm}$ and radius $0.15 \mathrm{~nm}$. Inside it contains four binding sites due to attractive dipolar carbonyl oxygen atoms (which exclude anions); with a fifth binding site at on its extra-cellular boundary. In contrast to graphene the charging paramater is large and on the order of the bulk hydration energy, $U_{c} \sim 160 k T$ (with $\epsilon_{w}^{*} \sim 9$ although it is likely to be smaller). This indicates strong interactions between the ions and the pore, resulting in highly coordinated motion with fluctuations between 2 and 3 ions in the filter. In the experimental set-up, symmetrical $\mathrm{KCl}$ solutions were used with concentrations ranging from $0.25-1.5 \mathrm{M}$, and currentvoltage curves were produced. To model this we reduce the state space and consider a two-level cation filter, with the

${ }^{2}$ Although we note that structural fluctuations are of order $0.075 \mathrm{~nm}$ [31]
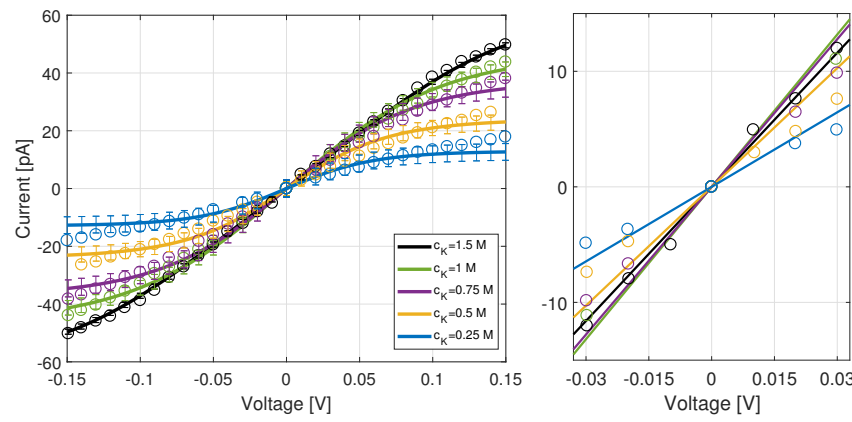

Fig. 5. (left) Theoretical fitting (solid) of Eq. (15) to data (circles) for KcsA [32] and the linear regime at low voltages (right). The diffusion coefficient was $0.2 D_{i}^{b}$, and the pore was symmetrical with $\left(\phi^{c} \sim \phi^{L} / 2\right)$. The energy barriers from mixing, global and local interactions for each concentration starting at $0.25 \mathrm{M}$ to $1.5 \mathrm{M}$ were: $3.6 k T, 3.68 k T, 3.94 k T$, $4.04 k T$, and $4.33 k T$ respectively.

results of fitting in Fig. 5. The theory produces a good fit to the data, although the current is slightly underestimated in the lower concentrations. The right panel contains the linear response calculated from equation (8) (with appropriate prefactor), which is equivalent of the linearised solution to the kinetic equations. Unlike the graphene pore, the filter characteristics were found to be symmetrical because the fraction of the voltage drop was $1 / 2$ and the energy barriers from mixing, local and global interactions were found to be monotonically increasing with concentration.

\section{CONCLUSIONS}

In summary, we have presented a multi-scale analysis of ionic conduction and selectivity through subnanometer pores. This includes a multi-species statistical theory describing the state space or occupancy of the pore. Our theory accounts for the properties of the pore such as its charge or geometry, the ionic composition of the surrounding solutions, the global interactions inside the pore, the local interactions specifically at the binding site, and any external driving forces. The important results from this theory have been: the explicit demonstration that noise (fluctuations) in the occupancy of the pore are directly proportional to the rate of conduction through the pore, and the derivation of optimal conducting parameters: the tuned fixed charge $n_{f}^{*}$ and the optimal chemical potential difference $\Delta \mu_{i}^{*}$. In addition we have extended the theory far from equilibrium with a set of master equations. Both theories provide excellent agreement when compared with the experimental and simulated data from the graphene nanopore and the biological KcsA channel.

We have also introduced a molecular dynamics investigation into the role of interactions during the permeation of ions through charged and neutral graphene pores. In both systems the geometry was comparable with a narrow pore. This narrow pore produces geometrical dehydration because water molecules are physically prevented from aligning around the ion as they do in the bulk reservoir (see [10]). Hence the fraction of water molecules lost can be calculated, and we find this to be in good agreement with our simulation data particularly for the uncharged pore. The small differences to the charged pore can be attributed to the lack of water-water and water-pore charge interactions. Consequently the effective continuum of water inside the pore must significantly 
differ to the bulk reservoir with a smaller effective dielectric coefficient $\epsilon_{w}^{*}$. We confirm this through comparisons of the PMFS and Coulomb's law.

To extend the applicability of our theory, we aim to explore the importance of coordinated motion which by describing it as the motion of a quasi-particle [16], [33], [34], with the expectation that it can lead to an improved continuous model of the current through the pore based on a coupled set of differential Chapman-Kolmogorov equations. In addition we would also like to note that the distribution functions (4) are related to the BBGKY hierarchy of kinetic equations [35]; and we plan to further explore this connection. Finally, we believe that the application of our methods will lead to new insights in both technology and biophysics.

\section{ACKNOWLEDGMENTS}

We are grateful to Robert Eisenberg, Olena Fedorenko, Igor Kaufman, Igor Khovanov, Stephen Roberts and Aneta Stefanovska for helpful discussions. The work was funded by a $\mathrm{PhD}$ Scholarship from the Faculty of Science and Technology of Lancaster University, the Engineering and Physical Sciences Research Council (grants EP/M016889/1 and EP/M015831/1), and by a Leverhulme Trust Research Project Grant RPG-2017-134. The authors declare no competing financial interest.

\section{REFERENCES}

[1] Z. Li, Y. Qiu, K. Li, J. Sha, T. Li, and Y. Chen, "Optimal design of graphene nanopores for seawater desalination," J. Chem. Phys., vol. 148, no. 1, p. 014703, Jan. 2018

[2] B. Hille, Ion Channels Of Excitable Membranes, 3rd ed. Sunderland, MA: Sinauer Associates, 2001.

[3] G. Eisenman, "On the elementary origin of equilibrium ion specificity," in Symposium on Membrane Transport and Metabolism, A. Kleinzelle and A. Kotyk, Eds. Academic Press, New York, 1961, pp. 163-179.

[4] B. Roux et al., "Ion selectivity in channels and transporters," J. Gen. Physiol., vol. 137, no. 5, pp. 415-426, 2011.

[5] R. C. Rollings, A. T. Kuan, and J. A. Golovchenko, "Ion selectivity of graphene nanopores," Nat. Commun., vol. 7, p. 11408, 2016.

[6] Z. He, J. Zhou, X. Lu, and B. Corry, "Bioinspired graphene nanopores with voltage-tunable ion selectivity for $\mathrm{Na}^{+}$and $\mathrm{K}^{+}, "$ ACS Nano, vol. 7, no. 11, pp. $10148-10157,2013$.

[7] Grossfield, A., "WHAM: The weighted Histogram Analysis method, version 2.0.9," 2017. [Online]. Available: http://membrane.urmc.rochester.edu/content/wham

[8] N. Kumar, D. Bouzida, R. Swendsen, P. Kollman, and J. Rosenberg, "The weighted histogram analysis method for free-energy calculations on biomolecules. I. the method," J. Comput. Chem., vol. 13, p. $10111021,1992$.

[9] S. Sahu and M. Zwolak, "Ionic selectivity and filtration from fragmented dehydration in multilayer graphene nanopores," Nanoscale, vol. 9, pp. 11424-11428, 2017.

[10] M. Zwolak, J. Lagerqvist, and M. Di Ventra, "Quantized ionic conductance in nanopores," Phys. Rev. Lett., vol. 103, p. 128102, 2009.

[11] L. D. Landau and E. M. Lifshitz, Statistical Physics. Course of Theoretical Physics. Volume 5. 3rd ed., Part I. Oxford: Pergamon Press, 1980.

[12] D. G. Luchinsky, W. A. T. Gibby, I. K. Kaufman, P. V. E. McClintock, and D. A. Timucin, "Relation between selectivity and conductivity in narrow ion channels," in 2017 International Conference on Noise and Fluctuations (ICNF), June 2017, pp. 1-4.

[13] D. G. Luchinsky, W. A. T. Gibby, I. Kaufman, D. A. Timucin, and P. V. E. McClintock, "Statistical theory of selectivity and conductivity in biological channels," ArXiv:1604.05758, 2016.

[14] E. Kitzing, "A novel model for saturation of ion conductivity in transmembrane channels," in Membrane Proteins: Structures, Interactions and Models: Proceedings of the Twenty-Fifth Jerusalem Symposium on Quantum Chemistry and Biochemistry Held in Jerusalem, Israel, May 18-21,1992, A. Pullman, J. Jortner, and B. Pullman, Eds. Dordrecht: Springer Netherlands, 1992, pp. 297-314.
[15] A. Kamenev, J. Zhang, A. I. Larkin, and B. I. Shklovskii, "Transport in one-dimensional Coulomb gases: From ion channels to nanopores," Physica A: Statistical Mechanics and its Applications, vol. 359, no. 1-4, pp. 129-161, 2006.

[16] V. N. Kharkyanen and S. O. Yesylevskyy, "Theory of single-file multiparticle diffusion in narrow pores," Phys. Rev. E, vol. 80, no. 3, pp. 1-19, 2009.

[17] I. K. Kaufman, D. G. Luchinsky, R. Tindjong, P. V. E. McClintock, and R. S. Eisenberg, "Multi-ion conduction bands in a simple model of calcium ion channels," Phys. Biol., vol. 10, no. 2, p. 026007, 2013.

[18] B. Roux, "Statistical Mechanical Equilibrium Theory of Selective Ion Channels," Biophys. J., vol. 77, no. 1, pp. 139-153, 1999.

[19] B. Roux, T. W. Allen, S. Berneche, and W. Im, "Theoretical and computational models of biological ion channels." Q. Rev. Biophys., vol. 37, no. 1, pp. 182-189, 2004.

[20] J. Zhang, A. Kamenev, and B. I. Shklovskii, "Conductance of ion channels and nanopores with charged walls: A toy model," Phys. Rev. Lett., vol. 95, p. 148101, 2005.

[21] C. W. J. Beenakker, "Theory of Coulomb-blockade oscillations in the conductance of a quantum dot," Phys. Rev. B, vol. 44, no. 4, pp. 16461656, 1991.

[22] Y. Alhassid, "Statistical theory of quantum dots," Rev. Mod. Phys., vol. 72, no. 4, pp. 895-968, 2000.

[23] D. Gillespie, W. Nonner, and R. S. Eisenberg, "Coupling PoissonNernst-Planck and density functional theory to calculate ion flux," $J$. Phys. Cond. Matt., vol. 14, no. 46, pp. 12 129-12 145, 2002.

[24] R. D. Coalson and M. G. Kurnikova, "Poisson-Nernst-Planck theory of ion permeation through biological channels," in Biological Membrane Ion Channels, S.-H. Chung, O. S. Andersen, and V. Krishnamurthy, Eds. New York: Springer, 2007, pp. 449-484.

[25] M. A. Wilson, T. H. Nguyen, and A. Pohorille, "Combining molecular dynamics and an electrodiffusion model to calculate ion channel conductance," J. Chem. Phys., vol. 141, no. 22, 2014.

[26] P. T. Landsberg, "Einstein and statistical thermodynamics. III. The diffusion-mobility relation in semiconductors," Eur. J. Phys., vol. 2, no. 4, p. 213, 1981.

[27] T. Becker, K. Nelissen, B. Cleuren, B. Partoens, and C. den Broeck, "Diffusion of interacting particles in discrete geometries: Equilibrium and dynamical properties," Phys. Rev. E, vol. 90, no. 5, p. 52139, Nov 2014.

[28] P. H. Nelson, "A permeation theory for single-file ion channels: Oneand two-step models," J. Chem. Phys., vol. 134, pp. 165 102-165 114, 2011.

[29] D. P. Tieleman, P. C. Biggin, G. R. Smith, and M. S. P. Sansom, "Simulation approaches to ion channel structure-function relationships," $Q$. Rev. Biophys., vol. 34, no. 4, pp. 473-561, 2001.

[30] D. Doyle et al., "The structure of the potassium channel: Molecular basis of $\mathrm{k}^{+}$conduction and selectivity," Science, vol. 280, no. 5360, pp. 69-77, 1998 .

[31] S. Y. Noskov, S. Berneche, and B. Roux, "Control of ion selectivity in potassium channels by electrostatic and dynamic properties of carbonyl ligands," Nature, vol. 431, no. 7010, p. 830, 2004.

[32] D. Meuser, H. Splitt, R. Wagner, and H. Schrempf, "Exploring the open pore of the potassium channel from streptomyces lividans," FEBS letters, vol. 462, no. 3, pp. 447-452, 1999.

[33] S. O. Yesylevskyy and V. N. Kharkyanen, "Quasi-particles in the selectivity filter can explain permeation in a channel with multiple occupancy," Phys. Chem. Chem. Phys., vol. 6, no. 12, p. 3111, 2004.

[34] _ "Barrier-less knock-on conduction in ion channels: peculiarity or general mechanism?" Chem. Phys., vol. 312, no. 1-3, pp. 127-133, 2005.

[35] N. N. Bogoliubov, "Kinetic Equations," J. Phys. USSR, vol. 10, p. 265274, 1946 